\title{
A Conversation Between Frans van Houten and Ken Herrmann
}

$\mathbf{K}$ en Herrmann, MD, MBA, a professor of nuclear medicine at the Universitätsklinikum Essen (Germany), talked with Frans van Houten, Chief Executive Officer (CEO) of Royal Philips Electronics. He studied economics at Erasmus University Rotterdam (The Netherlands), before beginning his career at Philips in 1986 in marketing and sales at Philips Data Systems. In 2002 he became codirector of the Philips consumer electronics division, and in 2004 CEO of Philips Semiconductors. He has been the CEO of Royal Philips since 2011 and is credited with revitalizing the business as a focused health technology company through targeted divestment and acquisition. He has also supported increased investment in research and development $(R \& D)$ in areas like digital pathology, medical wearables, and health care informatics. In 2017 he served as cochair at the World Economic Forum in Davos, Switzerland, and was one of the initiators of the international Compact for Responsive and Responsible Leadership, which aims to create a corporate governance framework with a focus on longterm sustainability of corporations and goals for society. He was named among the Top 20 Global Business Leaders in 2017 by Fortune magazine. He is also a member of the European Round Table of Industrialists, an advocacy organization including the 50 largest European multinational companies. He is a cofounder of NL2025, a platform of Dutch influencers who support initiatives to create a better future for The Netherlands.

Dr. Herrmann: Frans, thank you very much for taking the time. Everyone knows you as CEO of Philips, but what did you do before?

Mr. van Houten: I joined Philips in 1986 and started working on the informatics side, such as bank automation and high-speed telecommunication. Although that had little to do with health care, it laid the foundation for me to understand informatics, an area that is highly relevant for Philips's strategy today. Later I became CEO of the semiconductor division, which was sold in 2006 to private equity, and I moved with that divestiture. There I learned the perspective of private equity. In 2010 I was asked to come back to Philips to become the CEO in 2011.

Dr. Herrmann: Did your perspective on Philips change during your time at private equity?

Mr. van Houten: Looking at Philips from the outside changed my view significantly. I decided that the company had to change from a conglomerate to an operating company. We narrowed the focus on health technology, becoming a solutions partner as opposed to just making products. Here I am, 9 years into the job and a massive transformation of the company later. I am often asked: What is the motive? Why do you do things like that? For me, it is very important to give meaning. You can give meaning to your personal life and also to a company. We have succeeded in rejuvenating Philips into a leader in health technology, with $€ 20$ billion in revenue and growing profitability.

COPYRIGHT @ 2020 by the Society of Nuclear Medicine and Molecular Imaging. DOI: $10.2967 /$ jnumed.120.259754
Dr. Herrmann: What is your vision of a modern health technology company?

Mr. van Houten: The overarching assumption was that the value of a single product is diminishing and that there is an increasing value in a solutions approach to the patient journey. In theory, a healthy patient falls sick, needs to be diagnosed and treated, requires aftercare, and (hopefully) recovers to a healthy lifestyle. If not, the patient needs help living with a chronic disease. We need to optimize that jour-

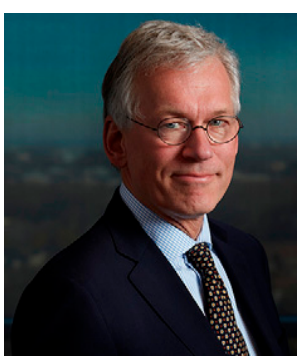

Frans van Houten ney. So if it is possible to prevent, we need to prevent. If you can diagnose early, we need to do this and treat appropriately. If you can treat these patients at home, treat them at home. There is a philosophy behind our strategy: we do not want to be a siloed player. We think there is more value in creating a platform approach to support patients and health care. Accordingly, we are looking at integration, combining systems, devices, software, and services. When translated to diagnostics, we know this is a multidisciplinary field. Precision medicine requires the integration of these multidisciplinary fields, such as radiology, nuclear medicine, pathology, and genomics. And what is the glue? The glue is data-informatics. The more that personalized medicine and therapies advance, the higher the need for precision diagnostics. Just improving the scanner or improving the image is not enough. It is the interpretation of the data from that image that is more important.

Dr. Herrmann: How do you apply this to diagnostics?

Mr. van Houten: We have built around our hardware a whole array of informatics packages with artificial intelligence (AI) being the driver, starting with how to run a radiology department (e.g., saving $30 \%$ of resources, optimizing scanning, and interpreting acquired data with automatic lesion detection, annotation, and size calculations). Then genomics information and digital pathology are added into the mix, allowing for digitized oncology tumor board meetings in which individual patients can be compared to cohorts. Even treatment pathways can be looked at and compared. We aim to offer the treating physician a comprehensive view and clinical decision support to eventually get to pathway selection for treatment.

Dr. Herrmann: Philips has strong roots in diagnostic imaging. How do you build the bridge to other modalities such as pathology?

Mr. van Houten: A part of our portfolio comes out of imaging and digital pathology. We aim to replace the microscope by digitizing tissue samples and analyzing these very high-resolution images, with which a much wider spectrum of cell composition is visible than with the human eye. The result is computational pathology, where tissue can be interpreted in a layered manner. We build the scanners, and we build the software that can handle both 
radiology and pathology, helping us to create integrated oncology diagnostic services.

Dr. Herrmann: You previously mentioned AI as the driver of innovation. How will AI contribute to diagnostics?

Mr. van Houten: AI can optimize operations, avoid mistakes, optimize settings, and so on. Then AI can dynamically adapt the way we scan, depending on what is being discovered during the scan, such as pathologic findings or even patient movement. This also includes operational enhancements, changing the protocols but also assisting the radiologist with image analysis: quantification, automatic annotation, automatic generation of action lists, follow-up actions, etc. Basically, it is clinical decision support supporting the radiologist to do a more effective and efficient job. We also use AI to do mission briefing for tumor boards for the oncologist, doing longitudinal studies, for example, of how a tumor has developed across multiple image acquisitions and across modalities. All of this saves time, supports a more comprehensive mission briefing, and then frees up the specialist's time to actually think about therapy and the patient.

Dr. Herrmann: PET and new PET tracers have been the key drivers of nuclear medicine diagnostics in the past. Where do you think nuclear medicine will be growing?

Mr. van Houten: Our R\&D colleagues tell us is that there is a lot of potential growth in new tracers like prostate-specific membrane antigen for prostate cancer and obviously amyloid and tau tracers for Alzheimer disease.
Mr. van Houten: The complexity of disease today means that no one is an expert in everything. An integration approach allows integration of know-how, education, and remote assistance. Why does the pathologist have to be on site? A specialized pathologist can look at the tissue sample from 100 miles away. By digital means experts can collaborate independently of their locations. Moreover, they can communicate with patients independently of location. We envision a cloud-enabled care system where scarceresource specialist doctors and patients who are remote can all become part of the coordinated care platform and where telehealth enables not only doctor-to-patient but also doctor-to-doctor dialog. That can overcome a lack of personnel.

Dr. Herrmann: Telemedicine is already well established in radiology, with nighthawks from India reading scans in the United States overnight. Will this be expanded to other areas in medicine?

Mr. van Houten: In China, Philips is enabling the remote programming of radiotherapy protocols, because some of the more rural hospitals do not have the onsite expertise. The next step is to facilitate a whole tele-oncology practice between rural and specialists' hospitals. We are also a leader in so-called eICU (electronic ICU) technologies, allowing for remote supervision of ICU beds. Think about infectious diseases - the fewer people who need to have access to the bed, the better. Through eICU all the interventionists can engage with the bedside remotely. In the United States we have hundreds of hospitals managing their ICU capacity at a safe distance, which is a clear upside in times such as COVID-19.

"It is also interesting that during COVID-19 we were able to make faster decisions- faster than ever."

Dr. Herrmann: You mentioned before that the importance of a single product is diminishing. This applies also to the hardware, the scanners. Something similar has been seen for coffee machines. When you look at Nespresso, they nowadays make money with the pods, the consumables. Could this be the PET business model of the future, with the consumables, the PET tracers, driving revenues?

Mr. van Houten: Theoretically, yes; but industries all have histories. The history of imaging is that it is not a combined business model. So it is not easy to escape your legacy. Could it be reinvented? Maybe. But that is not where we are today.

Dr. Herrmann: Rebuilding is more difficult than building new. What about areas in the world without legacy, such as China, India, or Africa?

Mr. van Houten: Let me take your question a little bit wider. Hospitals and care providers are becoming more open to new services because they have more pressure, more patients, and less money. There is a desire to shift toward a solutions approach. Philips is a technology partner to the health care system and can take care of the whole platform to interpret the data that are needed to bring it all together. Some care providers can do that themselves, but many have realized that they need a technology partner. As a consequence, our business model is shifting. I always wanted to shift from a transactional model to a partnering model taking coaccountability for productivity, improvement of clinical outcomes, etc. And I think society will want us to go in that direction.

Dr. Herrmann: An important part of the equation is welltrained personnel. There are many areas in the world where highly qualified personnel are not available.
Dr. Herrmann: COVID-19 is definitely fueling this process of digitalization and remote working. Shifting gears, you are also a board member of Novartis, one of the big players in the field of theranostics, which every nuclear medicine physician is excited about. Where do you see the role of Philips in theranostics?

Mr. van Houten: We are not entering the pharma world, but I do see diagnostic companies and therapy companies getting closer together. In theranostics you link diagnostics to the treatment selection and also the validation of this, whether the therapy is effective, closing the loop of measurement around the impact of the therapy. And by the way, not only to the theranostic field, I would say for any expensive drug treatment, society will demand that we close the loop on measurement of efficacy for the individual patient, which means that companion diagnostics will become the norm rather than the exception. Nuclear theranostics is a very exciting area, and I am very enthusiastic about being active in the oncology field.

Dr. Herrmann: You also mentioned PET, which is a substantial part of your portfolio. We have the feeling that PET is still underutilized. Is this something you would agree with? If so, how can we change that?

Mr. van Houten: That is an interesting question. Is it because the nuclear medicine department is separate from the radiology department?

Dr. Herrmann: Or is it rather because they are not separate? If nuclear medicine represents only a small part of an imaging department it is less independent, less proactive, and often less growing. 
Mr. van Houten: Definitely the linkage of precision diagnosing with therapy can give this field a boost. But you need to demonstrate clinical value and cost effectiveness. Reimbursement will follow a value model.

Dr. Herrmann: True, cost effectiveness is something that has to be addressed. Saving expenditures on expensive therapies with companion imaging could probably solve this issue quite quickly.

Mr. van Houten: Or at least ensuring that when you have an expensive therapy that it works and proving it. This is what I meant with "closing the loop" on the companion diagnostics. If evidence is there, I do not think that society will have a problem paying for the imaging.

Dr. Herrmann: Another exciting development is total-body PET, which is now offered by a vendor outside of the big 3 PET manufacturers. What are your thoughts about it?

Mr. van Houten: We are working on it with the University of Pennsylvania, so we are interested and excited. But it all comes back to the same point about value. We have to demonstrate that it is worth doing. What do you think?

Dr. Herrmann: Finding the right business model is the main challenge for this fantastic machine. I foresee 2 main applications. One is high throughput, meaning up to 100 patients per day per scanner. There are certain areas in the world like China that could easily fill such a scanner. But this also requires additional investment in infrastructure (more uptake rooms, etc.). Another application will be drug development, where many drugs fail, even as late as phase 2 or 3, when companies have already spent hundreds of millions of dollars. I think here the total-body PET can be very valuable, because almost any drug can be labeled with a PET radioisotope so that we could at least study pharmacokinetics, pharmacodynamics, etc. very early on.

Mr. van Houten: So that the mechanism of action can be verified.

Dr. Herrmann: Yes, but over all we are talking about 150, maybe 200, such scanners worldwide, compared to 5,000 and more for PET/CT. The market then obviously becomes quite difficult.

Mr. van Houten: For us, it is still in advanced development with the University of Pennsylvania, based on our digital PET/CT platform. We also have a large installed base in SPECT. I think that the world of SPECT is here to stay; therefore, through our partners, we are staying in that business.

Dr. Herrmann: If we believe in the success of theranostics, then SPECT might gain attractiveness, because a lot of therapy compounds can be easily imaged by SPECT but not with PET posttherapy. If dosimetry proves successful, SPECT will grow even stronger. Another question is about industry and academia relationships. A lot innovation comes from academia. Philips has a unique innovation hub in Eindhoven. What is your general impression on industry and academic collaboration and relationships?

Mr. van Houten: We absolutely need deep collaborations with academia to advance science, to advance clinical practice, to gain evidence, and eventually to also build the business case. In fact, we have an internal rule that we should not do any research without leading customer involvement, because otherwise we may be pursuing things that are too remote from the clinical world. We do that with leading universities across the world, in all fields, and also in nuclear medicine. I think about separating the research and the business parts, but I do not see that as an issue. I think the way of working has matured, and this is common practice.

Dr. Herrmann: Many companies also have scientists as board members, which I personally believe is crucial for success. However, some more rigid policies and rules prevent scientists from being board members. Do you think this is a loss of a symbiotic opportunity?
Mr. van Houten: For a company the size of Philips we use advisory boards to involve scientists. To be part of our global Philips board would most likely not be a satisfactory experience for scientists. Philips is an innovation company, and so we need conversations about innovation.

Dr. Herrmann: What is the most efficient way to innovate? Inhouse development? Acquisition? And then keeping startups at arm lengths or integration?

Mr. van Houten: We need to use all forms of innovation: in-house with deep research commitment, partnerships, acquiring startups, or taking stakes in companies. We have acquired 25 companies in the last 4 years. We have venture stakes in probably more than 100 startups.

Dr. Herrmann: Do you completely integrate the companies you acquire or keep them separate?

Mr. van Houten: I believe that eventually you have to integrate them, because otherwise why would you buy them? If it is a standalone company, there is no synergy and no return on investment. But we have to make sure that the agility and spirit of innovation are encouraged in the company rather than suppressed. I also do not have a problem keeping an acquisition more insulated for a certain time. I call it "ringfencing." But the longer-term benefit is in the integration. We also ringfence innovation programs internally at the company, where we want to ensure that we shield the nuggets through budgetary cycles or short-term pressures.

Dr. Herrmann: One of the biggest and unforeseen challenges is the COVID 19 pandemic. Is this only a crisis or also an opportunity?

Mr. van Houten: We were exposed to COVID-19 in January, because we have a large presence in China and our operations there were affected. We saw demand increase for diagnostic imaging, for patient monitors, and for ventilators. It allowed us to prepare and accelerate capacity for those modalities. We have seen a 4-5-fold increase in the demand for monitoring devices and ventilators. Ramping up supply chains in health care is not easy. We have mobilized hundreds and hundreds of extra people coming from our consumer health businesses to help with those acute care businesses. I have been personally on the phone with suppliers of semiconductor components and others to get more capacity. We have been on the phone with governments to get suppliers out of lockdown because we needed their capacity. It has been a very intensive and, to a degree, even exciting time. We have 80,000 people in the company and have launched the expression "triple duty of care." This means taking care of our customers and the patients served by our customers, taking care of the safety of our employees, and taking care of business continuity. We need to do all 3. Therefore, it is not an option to hunker down and try to escape. I know we have an obligation to our 6,000 field service engineers who have been going into hospitals with protective gear throughout the crisis. Our factory workers have been working in 3 shifts to ramp up production of monitoring devices and help in the factory and so on. It is also interesting that during COVID-19 we were able to make faster decisions - faster than ever. For us, this is actually rapid and agile decision-making time. You ask how we rethink that? It felt very good. We make a decision, move, and entrust people. We gain altitude and speed and do it because the world needs it. So, it is very gratifying.

Dr. Herrmann: The health care industry and supply chains have previously turned toward just-in-time delivery without extensive stocks. In many places in the world we were all suddenly running out of protective gear. Ramping up production is difficult, especially if it is beyond our control. Are we going to rethink a 
little bit how we do health care? Are we leaving the overarching pattern of more efficient, more economic and leaner operation, and turning back to local production and higher capacities?

Mr. van Houten: In normal times we produce 500 ventilators each week, and we ramped up production to 4,000 per week. You cannot maintain capacity at 4,000 per week just because somebody thought that they might need it. Likewise, I am often approached by governments, for example, the European Commission and White House, because everybody wants to have a factory in their own country and produce everything. But in health care, that is impossible, because the volumes are not big enough to justify it.

I have spent a lot of time arguing with governments to keep borders open, to avoid protectionism, to enable global supply chains to work, because even if you have a final assembly factory in one country, the components may still come from elsewhere. There are no semiconductor factories anymore in Europe; therefore, we are dependent on Asia. The world is interdependent, and we had better realize it. I have no problem with strategic reshoring-to a degree. But even when you do that, you cannot as a region be completely self-sufficient. But you can do something else: build stockpiles and have higher inventory levels of critical gear for pandemic-type situations. In business, we are used to scenario planning. If you do proper scenario planning, you can also anticipate that something can go wrong. So health care ministers should do scenario planning and realize that, for example, more ICU beds are needed. Germany, in fact, is better off because it had more ICU beds. Germany refused to take down the number of beds. As a consequence, The Netherlands had to send patients to Germany.

Health care governors and policymakers should think through their capacity needs and also the stockpiles required. You also have to maintain the stockpile. This again could be a public/ private partnership. Instead of having stuff sit in a warehouse and then discovering that it is old, industry could take care of that in a good collaboration. I think this is a better approach than having a factory in every country.
Dr. Herrmann: A lot of health systems took huge financial hits due to COVID-19, as also recently discussed in this column when Johannes Czernin interviewed Johnese Spisso, President of University of California at Los Angeles (UCLA) Health, CEO of the UCLA Hospital System, and Associate Vice Chancellor of UCLA Health Sciences (J Nucl Med. 2020;61[6]:777-779). Nevertheless, do you think that health care is one of the winners from COVID-19 because people now see the need for investment, ICU beds, etc.?

Mr. van Houten: Should we be optimistic or pessimistic? If you look at how the world learned from the severe acute respiratory syndrome (SARS) outbreak, then it was a temporary effect. But COVID-19 is bigger than SARS. I think the lessons learned will be more substantial. At the same time, the amount of money that society has available for health care is limited. Therefore, I think it is better to rethink health care so that we can do more with the same needs. One example would be segregating elective procedures from acute and infectious care-why would you need to do those in the same building? It is better to think about segregating these so that we would not have to completely stop elective procedures. Moreover, cloud technology can be employed (I mentioned the eICU as an example) to require fewer staff, manage more ICU beds remotely, and, aided by algorithms, look at how patients are trending. We can have more telehealth with remote patient support. We can have better health care data clouds.

I am glad that in Germany the discussion is now taking place about GAIA-X, a federated data infrastructure for Europe. Privacy is important, but privacy does not preclude us from having a proper federated data model so that we can manage outbreaks in a much better way and support the provisioning of care through the cloud rather than in physical proximity, where one can be infected. I hope that the COVID experience will help us reinvent the ways in which health care is being delivered.

Dr. Herrmann: You said you can be either optimistic or pessimistic. I clearly sense that you are optimistic. In that sense, I think it is a perfect finish. Thank you very much for your time. 